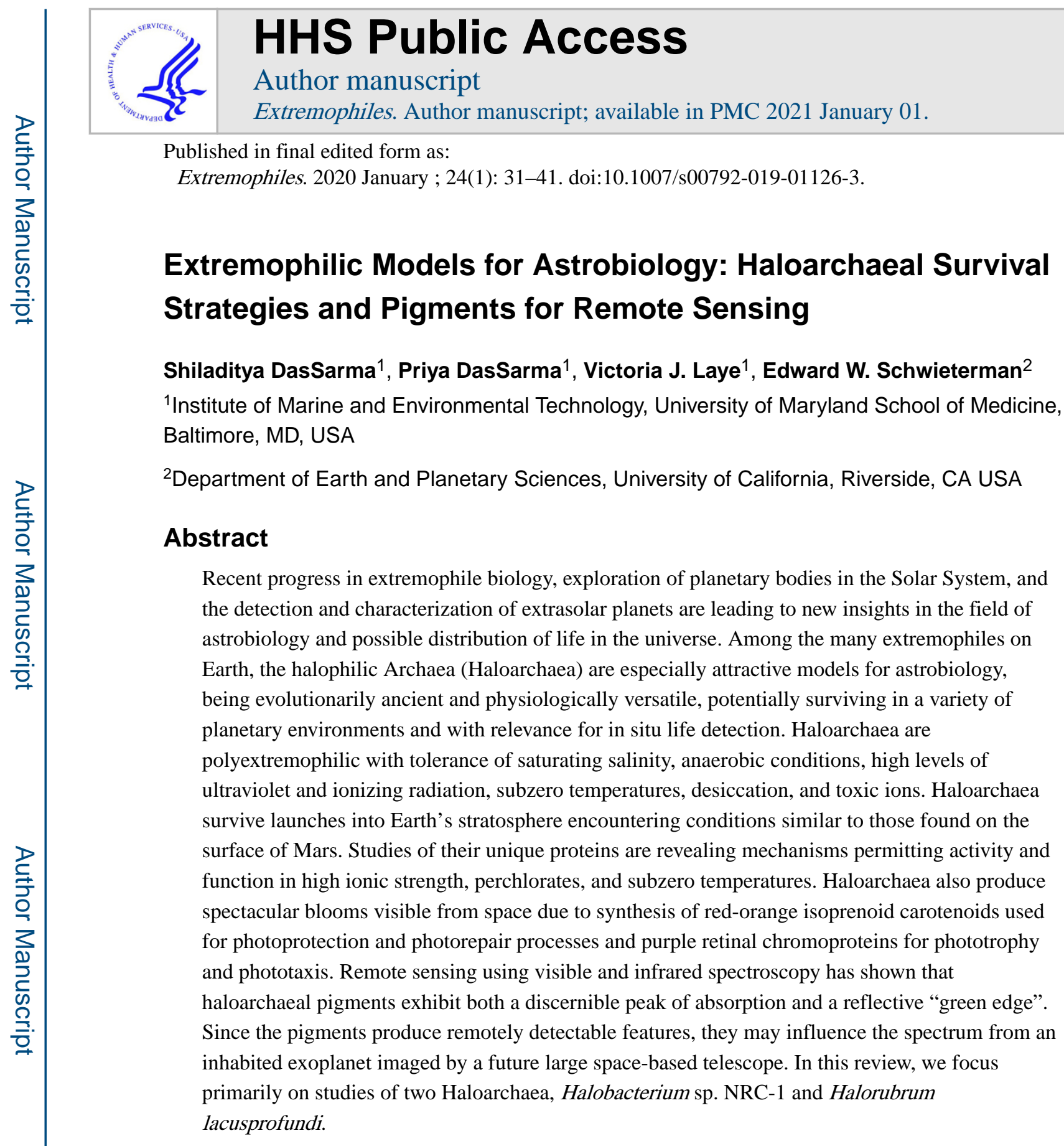

Keywords

Astrobiology; Biosignature; Haloarchaea; Phototrophy; Purple membrane; Retinal; Polyextremophile

\title{
Introduction
}

The emerging field of astrobiology addresses a fundamental philosophical question: 'Are we alone in the universe?' (Morrison 2001). Among the nearly 200 planets and satellites in the

“Corresponding author: Shiladitya DasSarma, sdassarma@som.umaryland.edu, Telephone: 1-410-234-8847, Fax: 1-410-234-8896. 
Solar System, and $\sim 4,000$ planets discovered so far in our galaxy, Earth remains the only known living world that can enlighten us about the potential answer (Lissauer, et al. 2014; Walker, et al. 2018). While common life-forms on Earth have traditionally informed us about the limits and capabilities of living organisms, the discovery of an ever-increasing variety of extremophiles over the past 50 years has expanded our appreciation of the physiological and biochemical capabilities of living organisms as well as illuminating the long history of evolution on our planet. Of special interest are extremophiles with the potential to survive in a variety of planetary environments within the solar system as well as the galaxy, which are relevant for in situ life detection.

Earth's extremophiles may be divided into two distinct categories: specialists, adapted to a single extreme; and generalists, adapted to multiple extremes (Rothschild and Mancinelli 2001). While considerable effort has gone into studies of specialists, such as hyperthermophiles and anaerobes, generalists like the halophilic Archaea (Haloarchaea) remain of significant interest for astrobiology since they survive multiple stressors, like those on the surface of Mars and potentially habitable exoplanets. Haloarchaea tolerate multiple extremes, forming dense cultures, and harbor light-capture pigments and phototrophic metabolism, which are potentially remotely detectable (DasSarma 2006). Consequently, Haloarchaea may influence the spectrum from an inhabited exoplanet, detectable from a future space telescope (Schwieterman, et al. 2018; DasSarma and Schwieterman 2019).

Many Haloarchaea are facultative aerobes with the ability to grow with or without oxygen, utilizing a variety of terminal electron acceptors, and in addition they have the capacity for phototrophic growth, making them physiologically unusually versatile microorganisms (DasSarma, et al. 2012;Kilic, et al. 2017;Mancinelli and Hochstein 1986;Martínez-Espinosa, et al. 2014;Müller and DasSarma 2005;Sorokin, et al. 2019;Sumper, et al. 1976).

Haloarchaea also are highly pigmented due to synthesis of carotenoids, resulting in bright blooms in hypersaline brine which may appear red, orange, or purple in color (DasSarma and Schwieterman 2019; de la Vega et al. 2016;Rodrigo-Baños et al. 2015; Yim et al. 2015). Coupled with our ability to isolate, construct, and characterize variants and mutants in the laboratory perturbed in their growth, pigmentation, and extremophilic properties, Haloarchaea offer the prospect of deeper insights into some fundamental questions in astrobiology.

\section{Evolution and habitability}

Our understanding of life's evolution on Earth is founded on knowledge of a common descent for all extant life, a concept supported by the existence of a universal genetic code (Söll and RajBhandary 2006). The ancestral life form from which all known life evolved is referred to as the last universal common ancestor (LUCA). Geochemical evidence of fossilized microorganisms in stromatolites suggests that LUCA appeared 3.7-3.8 billion years ago, when physical conditions were quite 'extreme' compared to conditions on the surface of Earth today (Fenchel 2002). As a result, the possibility that certain extremophilic species, e.g. early evolving Archaea, may resemble these early life forms has been postulated. However, extant species which are available for experimentation must have 
undergone considerable evolutionary changes during the billion years-long history of the planet and are likely to be quite different, as a result of processes such as lateral gene transfers (Kennedy, et al. 2001;Koonin 2015).

Recent studies of diverse extremophiles and their extreme environments on our planet nevertheless have offered the opportunity for better understanding the characteristics of life forms likely important for success on the early Earth (Merino, et al. 2019). They are also leading to insights into the limits to life at extremes of temperature, pressure, humidity, radiation and toxins. Extreme environments on Earth offer terrestrial analogs for understanding potential habitability of other planetary bodies. The ideal terrestrial microbial models for astrobiology should be capable of tolerating multiple extremes, like the Haloarchaea, which have evolved to survive in underground evaporites, as well as submarine brine pools beneath the sea, and in the upper fringes of the stratosphere above the protective ozone layer (DasSarma and DasSarma 2017; DasSarma and DasSarma 2018;DasSarma, et al. 2019; Grant, et al. 1998;Landis 2001).

\section{Models for astrobiology}

Among the great diversity of extremophiles known, Haloarchaea represent excellent models for astrobiology (DasSarma 2006,2007;DasSarma and DasSarma 2017). On Earth, they are frequently observed in buoyant, planktonic blooms above photosynthetic mats, which are similar to stratified microbial communities in ancient fossilized stromatolites (Noffke, et al. 2013). Haloarchaeal blooms are striking in color and offer the opportunity for direct visualization by remote sensing (Dalton, et al. 2009). These ancient microorganisms survive exposure to a variety of extreme conditions found on Mars, including desiccation, radiation, subzero temperatures, and the presence of perchlorate oxidizers (Table 1). They have also been reported to survive launches into the stratosphere and exposure to space conditions (DasSarma and DasSarma 2018;Horneck, et al. 2010).

Haloarchaea are members of the Domain Archaea, which likely evolved very early on Earth, possibly in the early Archaean (Fenchel 2002; DasSarma and Schwieterman 2019). The notion that Archaea appeared during the first billion years after formation of the planet is supported by the geochemical detection of isoprenoid compound derivatives used in their membrane lipids and pigments in ancient sediments. For example, such compounds have been detected in Isua, West Greenland sediments and dated to 3.7-3.8 billion years ago (Hahn and Haug 1986). The phototrophic and anaerobic metabolic capabilities of some Haloarchaea together with evidence for relatively recent acquisition of aerobic respiration by lateral gene transfer is consistent with such a scenario (Kennedy, et al. 2001;Koonin 2015).

Success of Haloarchaea on the Earth's surface in modern times is highlighted by highly pigmented blooms in widely distributed hypersaline solar salterns and lakes. Some haloarchaeal blooms, such as in south San Francisco Bay salterns or Great Salt Lake, Utah, have been observed by remote sensing from visible and infrared imaging sensors on airplanes (Dalton, et al. 2009) and visually by astronauts on the International Space Station (DasSarma, et al. 2016) (https://eol.jsc.nasa.gov/). The early evolution of retinal pigments has also been proposed to account for a "Purple Earth" (DasSarma and Schwieterman, 
2019). The combination of polyextremophily, detectability, and ancient evolution of Haloarchaea makes them attractive as astrobiological models among the extremophiles.

\section{Two haloarchaeal models}

Two Haloarchaea have been extensively studied as astrobiology models, Halobacterium sp. NRC-1, a highly pigmented buoyant species, isolated from the solar salterns of San Francisco Bay, California, USA, and H. lacusprofundi, a more brightly pigmented, biofilmforming species, isolated from the hypersaline Deep Lake, Antarctica (Anderson, et al. 2016;DasSarma 2007; DasSarma et al. 2019; Reid, et al. 2006). Halobacterium sp. NRC-1 is also distinguished by being the first among the Haloarchaea to have its genome sequenced (in 2000), an achievement that was followed by detailed postgenomic studies and characterization of the basis of survival under multiple extreme conditions (DasSarma 2004, 2006;Kennedy, et al. 2001;Ng, et al. 1998,2000;Peck et al. 2000).

A series of whole-genome transcriptomic studies on Halobacterium sp. NRC-1 addressed mechanisms of tolerance to high and low osmolarity, elevated and reduced temperatures, and UV and ionizing radiation (Coker, et al. 2007;Karan, et al. 2013,2014;McCready, et al. 2005). Genetic and transcriptomic studies explored the capability of the species to tolerate and grow microaerobically and anaerobically, phototrophically, and with heavy metal ions (DasSarma et al. 2012;Müller and DasSarma 2005;Wang, et al. 2004). A number of defining molecular characteristics were discovered, including core and signature genes, negatively charged proteins, expanded families of general transcription factors, and multiple origins and origin binding proteins (Capes, et al. 2011;Capes, et al. 2012). The Halobacterium sp. NRC-1 experimental system has provided considerable insights into the polyextremophilic characteristics of Haloarchaea (Figs. 1 and 2) (DasSarma, et al. 2006).

Several recent studies compared the mesophilic Halobacterium sp. NRC-1 to the coldadapted $H$. lacusprofundi for their tolerance to multiple stressors (Anderson, et al. 2016;DasSarma, et al. 2013; Reid, et al. 2006). Notable among them were survival following launches up to $35 \mathrm{~km}$ high in the atmosphere (DasSarma, et al. 2017). The cold-adapted species was better able to survive and grow after stratospheric launches and this property reflected better survival with freezing and thawing in the laboratory, and correlated to its more robust cold temperature response at genomic and transcriptomic levels.

An additional comparative study addressed the effects of exposure of these Haloarchaea to perchlorate, oxidizing ions that are common on the surface of Mars (Laye and DasSarma 2018). Interestingly, H. lacusprofundi was able to grow anaerobically using perchlorate as a terminal electron acceptor, providing a metabolism for potential survival on the red planet. In addition, $H$. lacusprofundi $\beta$-galactosidase enzyme was overexpressed and purified from Halobacterium sp. NRC-1 and found to function with either high concentrations of sodium or magnesium perchlorate, as well as allowing biological catalysis under current conditions on the surface of Mars (Laye and DasSarma 2018). 


\section{Acidic proteins}

Genomic analysis showed that Halobacterium sp. NRC-1, H. lacusprofundi, and other Haloarchaea have highly acidic proteomes (DasSarma and DasSarma 2015;Kennedy, et al. 2001) (Fig. 1). In contrast, nearly all other organisms have a near neutral proteome, with both acidic and basic proteins present. This difference likely reflects the unique adaptive strategy Haloarchaea use in their high salt environment, maintaining osmotic equilibrium with high internal salt concentrations, and balancing the sodium ions in their environment with potassium (DasSarma and DasSarma 2017). This "salt-in" strategy requires that haloarchaeal proteins are able to successfully compete for hydration and maintain solubility in the presence of high salt concentrations while retaining functionality (Karan, et al. 2012). Non-halophilic proteins generally lose function under high salt conditions due to loss of hydration, denaturation, aggregation, and precipitation by salting-out (Danson and Hough 1997).

\section{Bioinformatic analysis}

Genome-wide analysis of the first available haloarchaeal genome sequence for Halobacterium sp. NRC-1 led to the discovery of highly acidic, negatively charged proteins, with a unimodal isoelectric point distribution (pI 4.2), in contrast to the bimodal pI distributions seen for all other organisms (DasSarma and DasSarma 2015;Kennedy, et al. 2001). Amino acid composition analysis showed that there are nearly twice as many acidic glutamic and aspartic acid residues $(\sim 16 \%)$ in haloarchaeal proteins compared to basic arginine and lysine residues ( $\sim 8 \%)$. Glutamate residues in particular are known to have superior water-binding activity, and constitute the larger fraction, almost $9 \%$, in haloarchaeal proteins (Karan, et al. 2012).

Modeling and structural analysis of haloarchaeal proteins confirmed that acidic residues at the surface play a significant role in binding of essential water molecules and salt ions, preventing protein aggregation and providing flexibility (Kennedy, et al. 2001;Karan, et al. 2012). Over $80 \%$ of glutamic acid residues are located on surface of proteins and this single characteristic likely contributes to the ability of haloarchaeal proteins to maintain their protein hydration. Conversely, reduction in the salt concentration can result in charge repulsion between negatively charged proteins, leading to unfolding and loss of function (Karan, et al. 2012;Kennedy, et al. 2001). Additional protein mutagenesis studies confirmed the importance of acidic residues, including clusters of aspartate and glutamate residues on the surface (Esclapez, et al. 2007). Structural analysis showed the extended conformation of acidic residues pointed away from the protein surface, alternation in hydrophobic residues, and either burying of lysine side chains or their replacement by less hydrophilic arginine residues (Britton, et al. 2006;Karan, et al. 2012).

\section{Proteomic analysis}

Haloarchaea have been analyzed for encoded proteins that may be key to their adaptation to extreme environments using best reciprocal hits analysis to identify those which are conserved in the clade (Capes, et al. 2012) (Fig. 2). Among the nearly 800 conserved proteins, over 50 signature proteins were present in all the genomes included in the study 
and identified as potentially important to survival in the extreme environments in which Haloarchaea are found. While the function of most remains unknown, one of the Halobacterium sp. NRC-1 signature proteins, Ral, has been experimentally shown to function as likely accessory protein in double-stranded DNA break repair important for desiccation and radiation tolerance (DeVeaux, et al. 2007;Karan, et al. 2014).

The proteome of the Antarctic haloarchaeon, H. lacusprofundi, has been extensively studied by comparative genomic analysis (DasSarma, et al. 2013). After sequencing of the $H$. lacusprofundi genome, the proteome was found to be highly acidic, with an average $\mathrm{pI}$ of 4.6, and a high percentage (17\%) of acidic residues resulting from an increase in aspartic and glutamic acid residues (Anderson, et al. 2016). Amino acid composition of its predicted proteins was compared to other Haloarchaea by reciprocal blasting to address cold-tolerance of the proteins (DasSarma, et al. 2013). One H. lacusprofundi protein, a family $42 \beta$ galactosidase, which functions equally well in high concentrations of $\mathrm{KCl}$ and $\mathrm{NaCl}$, was used as a model enzyme for studying the necessary adaptations to low temperatures at high salinity (Karan, et al. 2013).

\section{Model polyextremophilic enzyme}

The remarkable polyextremophilic characteristics of $\beta$-galactosidase enzyme from $H$. lacusprofundi were studied biochemically and by mutagenesis, after overexpression and purification from Halobacterium sp. NRC-1 (Karan, et al. 2013). Its optimal activity was found to occur at a concentration of $4.5 \mathrm{M}$ salts in either $\mathrm{KCl}$ or $\mathrm{NaCl}$. It was also found to be active over a wide temperature range, from subzero temperatures where water remains liquid due to freezing point depression from the high salt concentrations, to temperatures as high as $70{ }^{\circ} \mathrm{C}$, with an optimum of $50^{\circ} \mathrm{C}$ (Karan, et al. 2013). The ability to measure enzyme activity at subzero temperatures in nearly saturated salinity made the $H$. lacusprofundi enzyme unique among this family of $\beta$-galactosidases.

H. lacusprofundi $\beta$-galactosidase activity was also characterized by steady-state kinetics with various concentrations of $\mathrm{MgCl}_{2}, \mathrm{NaClO}_{4}$, and $\mathrm{Mg}\left(\mathrm{ClO}_{4}\right)_{2}$, salts of considerable interest as a result of their detection on Mars (Hecht, et al. 2009). Magnesium acted as a weak competitive inhibitor $\left(\mathrm{K}_{\mathrm{I}}=0.3 \mathrm{M}\right)$, likely binding to the active site and blocking access, while $\mathrm{NaClO}_{4}$ acted as an even weaker noncompetitive inhibitor $\left(\mathrm{K}_{\mathrm{I}}=2 \mathrm{M}\right)$, consistent with binding elsewhere on the protein and causing conformational changes (Laye and DasSarma 2018). $\mathrm{Mg}\left(\mathrm{ClO}_{4}\right)_{2}$ acted as a stronger mixed inhibitor $\left(\mathrm{K}_{\mathrm{I}}=0.04 \mathrm{M}\right)$, exhibiting the combinatorial effects of both magnesium and perchlorate ions. Nevertheless, these studies and others aimed at growth inhibition showed that these Haloarchaea are able to tolerate concentrations of magnesium and perchlorate ions orders of magnitude higher than those expected on Mars (Laye and DasSarma 2018).

The $H$. lacusprofundi $\beta$-galactosidase enzyme was aligned to mesophilic and halophilic homologs to identify conserved residues that were diverged in the cold-active protein. In these related proteins of $\sim 800$ amino acid residues, only 6 residues were conserved in the mesophiles while being diverged in the cold-adapted species. These 6 residues were then mutated to determine whether they were responsible for conferring cold-adaptation to the protein (Laye, et al. 2017). Based on steady-state kinetic analysis, all 6 residues played a 
measurable role in conferring cold-adaptation to the enzyme and mutating them affected $\mathrm{K}_{\mathrm{m}}$, $\mathrm{k}_{\mathrm{cat}}$, or both, resulting in less cold-active enzymes. Molecular modeling showed small perturbations at the surface or in the interior of the protein, at considerable distances from the active site, with effects on both binding and catalytic efficiency.

\section{Pigment biosynthesis}

Most Haloarchaea such as Halobacterium spp. produce isoprenoid pigments that are important for their photobiology, functioning in photoprotection, photorepair, and phototrophic growth, and have the potential for astronomic detection (DasSarma, et al. 2001;DasSarma and Schwieterman 2019). These pigments have been studied since the 1970s by purification and biochemical characterization using chromatographic and spectroscopic methods, which has led to the deciphering of many steps in their biosynthetic pathway (Fig. 3) (Kushwaha et al. 1976). Understanding of this pathway has been further advanced by isolation of mutants (both natural and constructed) and analysis of biochemical and phenotypic traits coupled with phenotypic, biochemical, genetic, and genomic analyses.

\section{Carotenoids and retinal}

Haloarchaeal pigments include red-orange carotenoids, primarily C-50 bacterioruberins, characteristic of Halobacterium spp., C-40 lycopene and $\beta$-carotene, also common in plants, and retinal, a C-20 vitamin A aldehyde similar to visual pigments in higher organisms (Armstrong, et al. 1990;Kushwaha, et al. 1976;Sumper, et al. 1976). In the first step of the carotenoid pathway, two C-20 units are coupled to form the C-40 carotene phytoene by phytoene synthase. Phytoene is then converted to phytofluene, $\zeta$-carotene, and lycopene by the action of one or more phytoene desaturases.

The carotenoid pathway diverges at lycopene, with one branch producing $\beta$-carotene through the action of lycopene cyclase followed by synthesis of retinal from oxidative cleavage of $\beta$-carotene by $\beta$-carotene monooxygenase (Fig. 3 ). The other branch produces longer C-50 bacterioruberins by the addition of two C-5 units to lycopene by the action of lycopene elongase, followed by hydroxylation and reduction by one or more bacterioruberin hydroxylases or desaturases (Yang, et al. 2015). The bacterioruberins are the most abundant carotenoids produced in Haloarchaea and have been found to be involved in photoprotection and photorepair of DNA damage from UV radiation, while retinal is produced in sufficient quantities to serve as a chromophore for opsins used in phototrophy and phototaxis (DasSarma, et al. 2001;McCready 1996;Stoeckenius and Bogomolni 1982).

Carotenoid biosynthesis has been characterized genetically in Halobacterium sp. NRC-1 and similar species. Studies established the conversion of lycopene to $\beta$-carotene followed by oxidative cleavage of $\beta$-carotene to retinal, as well as the elongation of lycopene to bisanhydrobacterioruberin and its hydration to a variety of bacterioruberins (Fang, et al. 2010; Yang, et al. 2015). Mutants lacking bacterioruberins have been reported, occurring spontaneously by insertions of ISH elements in genes coding lycopene elongase (Dummer, et al. 2011). 


\section{Purple membrane}

Haloarchaea contain retinal proteins that bind covalently in a 1:1 ratio via a Schiff's base linkage to lysine (Bayley, et al. 1981). The most abundant retinal proteins is bacteriorhodopsin (Fig. 4), a light-driven proton pump that forms a two-dimensional crystalline lattice of trimers in the purple membrane, a specialized region of the cell membrane (Stoeckenius and Bogomolni 1982). Depending on the abundance of purple membrane in cells and the light intensity in the environment, a period of phototrophic growth may be supported (Sumper, et al. 1976). The purple membrane can be isolated by sucrose gradient centrifugation, and has been extensively studied for its functional characteristics (Krebs and Khorana 1993). The genes for purple membrane synthesis have been studied by transcriptomic analysis and isolation of mutants of the opsin or chromophore (DasSarma, et al. 2012).

Mutants in the pathway include orange-colored variants that were the result of defects in purple membrane synthesis as well as those which were deep purple from over-production of purple membrane (DasSarma 1989). The bacterio-opsin (bop) gene was shown to be frequently subject to insertion by ISH elements and led to the discovery and analysis of additional upstream genes in the regulon (Yang, et al. 1996;Baliga, et al. 2001). They include $b a t$, a regulatory gene, deletion of which resulted in elimination of purple membrane production without inhibiting the synthesis of bacterioruberins (DasSarma, et al. 2012).

Mutants defective in retinal synthesis were also isolated and studied. Deletions in two genes, brp and blh, coding for $\beta$-carotene monooxygenases, which cleave $\beta$-carotene into two molecules of retinal, blocks the final step in retinal synthesis (Peck, et al. 2001). Deletion of brp resulted in decreased bacteriorhodopsin and retinal levels, while bacterio-opsin levels were normal, and $\beta$-carotene levels were increased, while deletion of blh resulted in reduced bacteriorhodopsin accumulation. In a double-deletion mutant ( $\Delta b r p \Delta b l h)$ there was no detectable level of bacteriorhodopsin or retinal, without changes in bacterio-opsin accumulation while $\beta$-carotene levels were increased. The two genes, brp and blh, are also likely differentially regulated, with the brp gene located directly upstream of the bop gene and the blh gene located in a gene cluster with $\beta$-carotene cyclase and subunits of ATP synthase (DasSarma, et al. 2012;Peck, et al. 2001). Finally, the cytochrome P450 monooxygenase was also implicated in carotenoid biosynthesis, deletion of which increased bacterioruberin while decreasing purple membrane content (Müller, et al. 2019).

\section{Astronomical biosignatures}

Surface features indicative of living biomass, including haloarchaeal pigments, have been proposed as potential astronomical biosignatures (Schwieterman, et al. 2018;Seager, et al. 2005). On the modern Earth, chlorophyll pigments produce a reflective spectroscopic edge, i.e., the "red edge" at $\sim 700 \mathrm{~nm}$, where vegetation transitions from primarily absorbing visible light to reflecting and scattering near-infrared light (Gates, et al. 1965;Knipling 1970). A wide variety of non-photosynthetic organisms, including phototrophic microorganisms, have pigments that also produce "edge" wavelengths in reflectance spectra and could conceivably be remote signatures if the biomass is sufficiently well-distributed 
over a planetary surface (Hegde, et al. 2015;Lingam and Loeb 2017; Schwieterman, et al. 2015).

Among potential astronomical biosignatures, haloarchaeal pigments, which may have predated photosynthetic pigments, are attractive candidates (Schwieterman, et al.

2018; DasSarma and Schwieterman 2019). Brine lakes with pigmented blooms of diverse Haloarchaea are widely distributed and represent potential analogs for remote life detection on other planets. One of the largest such lakes, Great Salt Lake in the United States Great Basin, extends over ca. 4,000 square kilometers, with blooms of halophilic microorganisms observed from space (DasSarma, et al. 2016). In culture, Halobacterium sp. NRC-1 exhibits pink floating biomass due to cell buoyancy and refraction of light by hollow gas vesicle nanoparticle organelles as well as high quantities of carotenoid pigments and purple membrane (DasSarma, et al. 2006;DasSarma 2007). H. lacusprofundi cultures produce even higher levels of carotenoid pigments than Halobacterium sp. NRC-1, and appear bright orange.

Haloarchaeal pigments have been remotely detected in the hypersaline solar salterns of South San Francisco Bay used for mining sea salt. These blooms are readily observed from airplanes and can take on different hues, including orange, red, or purple, at different times. The Jet Propulsion Lab generated 10-year record of remote spectral information using an airborne visible and infrared imaging spectrophotometer (Dalton, et al. 2009). The strongest reflection spectral signals were seen in the most saline ponds (>200 g/L), where carotenoid and bacteriorhodopsin peaks (reflectance troughs) were observed at 500-570 nm. At lower salinities $(<50 \mathrm{~g} / \mathrm{L})$, chlorophyll absorption spectra were most readily visible.

\section{Future studies}

The science of exoplanets is rapidly moving from detection and bulk characterization to atmospheric spectroscopy and studies of chemical make-up. While current technology limits the study of Earth-size planets in the habitable zones of nearby stars, futur-espace-based telescope concepts such as LUVOIR or HabEx would have the capacity to directly image these worlds and characterize their atmospheres and surfaces at visible wavelengths (Bolcar, et al. 2017; Roberge and Moustakas 2018). To better inform the design and implementation of these missions, astrobiologists have worked to catalog the ensemble of potentially observable biosignatures - remote spectral or photometric indicators of life (Schwieterman, et al. 2018).

Haloarchaea and other retinal-based phototrophs (DasSarma and DasSarma 2017;Finkel, et al. 2013) produce a spectral signature different than, but complementary to vegetation. In contrast to photosynthetic vegetation and their 700-nm red-edge, laboratory studies of $H$. lacusprofundi produce a $~ 550 \mathrm{~nm}$ "green-edge" (Fig. 5) (DasSarma and Schwieterman 2019). Modeling suggests that blooms of such Haloarchaea suspended in an evaporite solution, including radiative effects from the scattering and absorption of water, combined with the increasing microbial reflectivity at green, yellow, and red wavelengths, would produce a peak reflection at $\sim 680 \mathrm{~nm}$ (Fig. 5). This wavelength is relatively transparent to atmospheric absorption and scattering. Given sufficient surface coverage, this pigment 
signature may be detectable in the overall planetary spectrum of an exoplanet

(Schwieterman, et al. 2015).

The use of Haloarchaea and other retinal phototrophs for a remote astronomical biosignature is specially promising because these microorganisms can acquire light energy for metabolism and are known empirically to produce macroscopic and remotely observable blooms on Earth (Dalton, et al. 2009; DasSarma 2006, 2007;DasSarma, et al. 2016). Moreover, the Purple Earth hypothesis suggests that planets may evolve through a period when these pigments may dominate and therefore may represent an early stage of evolution (DasSarma and Schwieterman 2019). If the prevailing conditions on an exoplanet are similar, it is possible that Haloarchaea-like signatures may be remotely observable.

Fortunately, visible wavelengths are likely to be included in future space and ground-based observatories capable of imaging planets in their habitable zones (Fujii, et al. 2018), and so should detect sufficiently strong "edge" biosignatures. It should be noted, however, that the signal-to-noise requirements may be more challenging compared to, for example, detecting molecular oxygen in the atmosphere (Brandt and Spiegel 2014). Future measurements are needed to thoroughly survey the reflectance spectra of Haloarchaea and other retinal phototrophs in vivo and in solution and enumerate potential false positives from abiotic surfaces, such as minerals (e.g., Clark, et al. 2007). Additional modeling efforts are also essential to ascertain the minimum surface coverage and cell density required to detect these surface biosignatures on nearby exoplanets, given the likely capabilities of future space telescopes.

\section{Concluding remarks}

Haloarchaea represent outstanding extremophiles for astrobiology studies since they survive in a variety of planetary environments and are relevant for in situ life detection. Their evolutionary, physiological, and spectroscopic properties make them of interest when considering the origin of life, biochemical and metabolic capabilities, and pigments that may serve as potential remote biosignatures. Future laboratory and modeling studies of these extremophiles together with large space-based telescopes that are planned for future astronomical observations are likely to lead to new avenues of highly consequential research, with the potential for answering questions about the rarity or ubiquity of life in the universe.

\section{Acknowledgements}

Exobiology research in the S.D. laboratory is supported by National Aeronautics and Space Administration (NASA) grants NNX15AM07G and NNH18ZDA001N and biomedical research is supported by National Institutes of Health (NIH) grant R21 AI139808. E.S. is supported by a NASA Postdoctoral Program Fellowship, administered by the Universities Space Research Association, and by the NASA Astrobiology Institute's Alternative Earths and Virtual Planetary Laboratory teams under Cooperative Agreement Nos. NNA15BB03A and NNA13AA93A, respectively. The Virtual Planetary Laboratory is also supported by the NASA Astrobiology Program under grant number 80NSSC18K0829.

\section{Abbreviations}

UV ultraviolet 


$\begin{array}{ll}\text { LUCA } & \text { Last universal common ancestor } \\ \text { pI } & \text { Isoelectric point } \\ \text { HOG } & \text { Haloarchaeal orthologous groups } \\ \text { BR } & \text { Bacteriorhodopsin }\end{array}$

\section{References}

Anderson IJ, DasSarma P, Lucas S, Copeland A, Lapidus A, Del Rio TG, Tice H, Dalin E, Bruce DC, Goodwin L, Pitluck S, Sims D, Brettin TS, Detter JC, Han CS, Larimer F, Hauser L, Land M, Ivanova N, Richardson P, Cavicchioli R, DasSarma S, Woese CR and Kyrpides NC (2016) Complete genome sequence of the Antarctic Halorubrum lacusprofundi type strain ACAM 34. Standards in Genomic Sciences 11:10.1186/s40793-016-0194-2

Armstrong GA, Alberti M and Hearst JE (1990) Conserved enzymes mediate the early reactions of carotenoid biosynthesis in nonphotosynthetic and photosynthetic prokaryotes. Proceedings of the National Academy of Sciences 87:9975-9979.

Baliga NS, Kennedy SP, Ng WV, Hood L and DasSarma S (2001) Genomic and genetic dissection of an archaeal regulon. Proceedings of the National Academy of Sciences 98:2521-2525.

Bayley H, Huang K-S, Radhakrishnan R, Ross AH, Takagaki Y and Khorana HG (1981) Site of attachment of retinal in bacteriorhodopsin. Proceedings of the National Academy of Sciences 78:2225-2229.

Bolcar MR, Aloezos S, Bly VT, Collins C, Crooke J, Dressing CD, Fantano L, Feinberg LD, France K, Gochar G, Gong Q, Hylan JE, Jones A., Linares I, Postman M, Pueyo L, Roberge A, Sacks L, Tompkins S, West G (2017) The large UV/optical/infrared surveyor (LUVOIR): decadal mission concept design update. In: MacEwen HA, Breckinridge JB (eds) Proceedings Volume 10398, UV/ Optical/IR space telescopes and instruments: innovative technologies and concepts VIII. Society of Photo-Optical Instrumentation Engineers Optical Engineering + Applications 2017, pp 9 https://doi: 10.1117/12.2273848

Brandt TD and Spiegel DS (2014) Prospects for detecting oxygen, water, and chlorophyll on an exoEarth. Proceedings of the National Academy of Sciences 111:13278-13283.

Britton KL, Baker PJ, Fisher M, Ruzheinikov S, Gilmour DJ, Bonete M-J, Ferrer J, Pire C, Esclapez J and Rice DW (2006) Analysis of protein solvent interactions in glucose dehydrogenase from the extreme halophile Haloferax mediterranei. Proceedings of the National Academy of Sciences 103:4846-4851.

Capes MD, Coker JA, Gessler R, Grinblat-Huse V, DasSarma SL, Jacob CG, Kim J-M, DasSarma P and DasSarma S (2011) The information transfer system of halophilic archaea. Plasmid 65:77-101. [PubMed: 21094181]

Capes MD, DasSarma P and DasSarma S (2012) The core and unique proteins of haloarchaea. BMC genomics 13:39. [PubMed: 22272718]

Clark RN, Swayze GA, Wise R, Livo KE, Hoefen T, Kokaly RF and Sutley SJ (2007) USGS digital spectral library splib06a. US geological survey, digital data series 231:2007.

Coker JA, DasSarma P, Kumar J, Müller JA and DasSarma S (2007) Transcriptional profiling of the model Archaeon Halobacterium sp. NRC-1: responses to changes in salinity and temperature. Saline Systems 3:6. [PubMed: 17651475]

Dalton J, Palmer-Moloney L, Rogoff D, Hlavka C and Duncan C (2009) Remote monitoring of hypersaline environments in San Francisco Bay, CA, USA. International Journal of Remote Sensing 30:2933-2949.

Danson MJ and Hough DW (1997) The structural basis of protein halophilicity. Comparative Biochemistry and Physiology Part A: Physiology 117:307-312.

DasSarma P, Zamora RC, Müller JA and DasSarma S (2012) Genome-wide responses of the model archaeon Halobacterium sp. strain NRC-1 to oxygen limitation. Journal of Bacteriology 194:55305537. [PubMed: 22865851] 
DasSarma P, Laye VJ, Harvey J, Reid C, Shultz J, Yarborough A, Lamb A, Koske-Phillips A, Herbst A, Molina F, Grah O, Phillips T and DasSarma S (2017) Survival of halophilic Archaea in Earth's cold stratosphere. International Journal of Astrobiology 16:321-327.

DasSarma P, Capes MD and DasSarma S (2019) Comparative Genomics of Halobacterium Strains from Diverse Locations In Microbial Diversity in the Genomic Era, Das S, Dash HR 1st Edition, pp. 285-322, Academic Press.

DasSarma P, Tuel K, Nierenberg SD, Phillips T, Pecher WT, and DasSarma S (2016) Inquiry-driven Teaching \& Learning Using the Archaeal Microorganism Halobacterium NRC-1. The American Biology Teacher 78:71-3.

DasSarma S (1989) Mechanisms of genetic variability in Halobacterium halobium: the purple membrane and gas vesicle mutations. Canadian Journal of Microbiology 35:65-72. [PubMed: 2541878]

DasSarma S, Kennedy SP, Berquist B, Ng WV, Baliga NS, Spudich JL, Krebs MP, Eisen JA, Johnson $\mathrm{CH}$ and Hood L (2001) Genomic perspective on the photobiology of Halobacterium species NRC-1, a phototrophic, phototactic, and UV-tolerant haloarchaeon. Photosynthesis Research 70:317. [PubMed: 16228359]

DasSarma S (2004) Genome sequence of an extremely halophilic archaeon In: Fraser C, Read T and Nelson KE (ed) Microbial Genomes C.M. Humana Press, Inc Totowa, NJ pp 383-399.

DasSarma S (2006) Extreme halophiles are models for astrobiology. Microbe-American Society for Microbiology 1:120-126.

DasSarma S, Berquist BR, Coker JA, DasSarma P and Müller JA (2006) Post-genomics of the model haloarchaeon Halobacterium sp. NRC-1. Saline Systems 2:3. [PubMed: 16542428]

DasSarma S (2007) Extreme Microbes. American Scientist 95:224-231.

DasSarma S, Capes M and DasSarma P (2009) Haloarchaeal megaplasmids In: Schwartz E (ed) Microbial megaplasmids Springer pp 3-30. doi:10.1007/7171_2008_17

DasSarma S, Capes MD, Karan R and DasSarma P (2013) Amino acid substitutions in cold-adapted proteins from Halorubrum lacusprofundi, an extremely halophilic microbe from Antarctica. PLoS One 8:e58587.

DasSarma S and DasSarma P (2015) Halophiles and their enzymes: negativity put to good use. Current Opinion in Microbiology 25:120-126. [PubMed: 26066288]

DasSarma S and DasSarma P (2017) Halophiles In: Encyclopedia of Life Sciences, John Wiley \& Sons, Ltd Chichester DOI: 10.1002/9780470015902.a0000394.pub4

DasSarma S and DasSarma P (2018) Survival of microbes in Earth's stratosphere. Current Opinion in Microbiology 24-30. 10.1016/j.mib.2017.11.002. [PubMed: 29156444]

DasSarma S and Schwieterman EW (2019) Early evolution of purple retinal pigments on Earth and implications for exoplanet biosignatures. International Journal of Astrobiology 10.1017/ S1473550418000423

de la Vega M, Sayago A, Ariza J, Barneto AG and León R (2016) Characterization of a bacterioruberin-producing Haloarchaea isolated from the marshlands of the Odiel river in the southwest of Spain. Biotechnol Prog. 32:592-600. doi:10.1002/btpr.2248. [PubMed: 26871874]

DeVeaux LC, Müller JA, Smith J, Petrisko J, Wells DP and DasSarma S (2007) Extremely radiationresistant mutants of a halophilic archaeon with increased single-stranded DNA-binding protein (RPA) gene expression. Radiation Research 168:507-514. [PubMed: 17903038]

Dummer AM, Bonsall JC, Cihla JB, Lawry SM, Johnson GC and Peck RF (2011) Bacterioopsinmediated regulation of bacterioruberin biosynthesis in Halobacterium salinarum. Journal of Bacteriology JB. 05376-05311.

Esclapez J, Pire C, Bautista V, Martínez-Espinosa R, Ferrer J and Bonete M (2007) Analysis of acidic surface of Haloferax mediterranei glucose dehydrogenase by site-directed mutagenesis. FEBS Letters 581:837-842. [PubMed: 17289028]

Fang C-J, Ku K-L, Lee M-H and Su N-W (2010) Influence of nutritive factors on C50 carotenoids production by Haloferax mediterranei ATCC 33500 with two-stage cultivation. Bioresource technology 101:6487-6493. [PubMed: 20362434]

Fenchel T (2002) The origin and early evolution of life. Oxford University Press, USA 
Finkel OM, Béja O and Belkin S (2013) Global abundance of microbial rhodopsins. The ISME journal 7:448. [PubMed: 23051692]

Fujii Y, Angerhausen D, Deitrick R, Domagal-Goldman S, Grenfell JL, Hori Y, Kane SR, Pallé E, Rauer H and Siegler N (2018) Exoplanet biosignatures: observational prospects. Astrobiology 18:739-778. [PubMed: 29938537]

Gates DM, Keegan HJ, Schleter JC and Weidner VR (1965) Spectral properties of plants. Applied Optics 4:11-20.

Grant WD, Gemmell RT and McGenity TJ (1998) Halobacteria: the evidence for longevity. Extremophiles 2:279-287. [PubMed: 9783175]

Hahn J and Haug P (1986) Traces of Archaebacteria in ancient sediments. Systematic and Applied Microbiology 7:178-183.

Hecht M, Kounaves S, Quinn R, West S, Young S, Ming D, Catling D, Clark B, Boynton W, Hoffman J, DeFlores LP, Gospodinova K, Kapit J and Smith PH (2009) Detection of perchlorate and the soluble chemistry of martian soil at the Phoenix lander site. Science 325:64-67. [PubMed: 19574385]

Hegde S, Paulino-Lima IG, Kent R, Kaltenegger L and Rothschild L (2015) Surface biosignatures of exo-Earths: remote detection of extraterrestrial life. Proceedings of the National Academy of Sciences 112:3886-3891.

Horneck G, Klaus DM and Mancinelli RL (2010) Space microbiology. Microbiology and Molecular Biology Reviews 74:121-156. [PubMed: 20197502]

Karan R, Capes MD and DasSarma S (2012) Function and biotechnology of extremophilic enzymes in low water activity. Aquatic Biosystems 8:4. [PubMed: 22480329]

Karan R, Capes MD, DasSarma P and DasSarma S (2013) Cloning, overexpression, purification, and characterization of a polyextremophilic $\beta$-galactosidase from the Antarctic haloarchaeon Halorubrum lacusprofundi. BMC Biotechnology 13:1. [PubMed: 23281894]

Karan R, DasSarma P, Balcer-Kubiczek E, Weng RR, Liao C-C, Goodlett DR, Ng WV and DasSarma $S$ (2014) Bioengineering radioresistance by overproduction of RPA, a mammalian-type singlestranded DNA-binding protein, in a halophilic archaeon. Applied Microbiology and Biotechnology 98:1737-1747. [PubMed: 24292079]

Kennedy SP, Ng WV, Salzberg SL, Hood L and DasSarma S (2001) Understanding the adaptation of Halobacterium species NRC-1 to its extreme environment through computational analysis of its genome sequence. Genome Research 11:1641-1650. [PubMed: 11591641]

Kilic V, Kilic GA, Kutlu HM and Martínez-Espinosa RM (2017) Nitrate reduction in Haloferax alexandrinus: the case of assimilatory nitrate reductase. Extremophiles. 21:551-561. doi: 10.1007/ s00792-017-0924-4. [PubMed: 28321615]

Knipling EB (1970) Physical and physiological basis for the reflectance of visible and near-infrared radiation from vegetation. Remote Sensing of Environment 1:155-159.

Koonin EV (2015) Origin of eukaryotes from within archaea, archaeal eukaryome and bursts of gene gain: eukaryogenesis just made easier? Philos Trans R Soc Lond B Biol Sci. 370:20140333. doi: 10.1098/rstb.2014.0333. [PubMed: 26323764]

Krebs MP and Khorana HG (1993) Mechanism of light-dependent proton translocation by bacteriorhodopsin. Journal of Bacteriology 175:1555-1560. [PubMed: 8383660]

Kushwaha SC, Kates M and Porter JW (1976) Enzymatic synthesis of C40 carotenes by cell-free preparation from Halobacterium cutirubrum. Canadian Journal of Biochemistry 54:816-823. [PubMed: 971465]

Landis GA (2001) Martian Water: Are There Extant Halobacteria on Mars? Astrobiology 1:161-164. [PubMed: 12467119]

Laye VJ, Karan R, Kim J-M, Pecher WT, DasSarma P and DasSarma S (2017) Key amino acid residues conferring enhanced enzyme activity at cold temperatures in an Antarctic polyextremophilic $\beta$-galactosidase. Proceedings of the National Academy of Sciences 114:12530 12535. 10.1073/pnas.1711542114

Laye VJ and DasSarma S (2018) An Antarctic extreme halophile and its polyextremophilic enzyme: Effects of perchlorate salts. Astrobiology 18:10.1017/S1473550416000410 
Lingam M and Loeb A (2017) Natural and artificial spectral edges in exoplanets. Monthly Notices of the Royal Astronomical Society: Letters

Lissauer JJ, Dawson RI and Tremaine S (2014) Advances in exoplanet science from Kepler. Nature 513:336. [PubMed: 25230655]

Mancinelli RL and Hochstein LI (1986) The occurrence of denitrification in extremely halophilic bacteria. FEMS Microbiology Letters 35:55-58. [PubMed: 11542048]

Martínez-Espinosa RM, Richardson DJ and Bonete MJ (2014) Characterisation of chlorate reduction in the haloarchaeon Haloferax mediterranei. Biochim Biophys Acta. 1850:587-594. doi: 10.1016/ j.bbagen.2014.12.011. [PubMed: 25512066]

McCready S (1996) The repair of ultraviolet light-induced DNA damage in the halophilic archaebacteria, Halobacterium cutirubrum, Halobacterium halobium and Haloferax volcanii. Mutation Research/DNA Repair 364:25-32.

McCready S, Müller JA, Boubriak I, Berquist BR, Ng WL and DasSarma S (2005) UV irradiation induces homologous recombination genes in the model archaeon, Halobacterium sp. NRC-1. Saline Systems 1:3. [PubMed: 16176594]

Merino N, Aronson HS, Bojanova DP, Feyhl-Buska J, Wong ML, Zhang S and Giovannelli D (2019) Living at the Extremes: Extremophiles and the Limits of Life in a Planetary Context. Front Microbiol. 10:780. doi: 10.3389/fmicb.2019.00780. [PubMed: 31037068]

Morrison D (2001) The NASA astrobiology program. Astrobiology 1:3-13. [PubMed: 12448992]

Müller JA and DasSarma S (2005) Genomic analysis of anaerobic respiration in the archaeon Halobacterium sp. strain NRC-1: dimethyl sulfoxide and trimethylamine N-oxide as terminal electron acceptors. Journal of Bacteriology 187:1659-1667. [PubMed: 15716436]

Müller WJ, Smit MS, van Heerden E, Capes MD and DasSarma S (2019) Complex Effects of Cytochrome P450 Monooxygenase on Purple Membrane and Bacterioruberin Production in an Extremely Halophilic Archaeon: Genetic, Phenotypic, and Transcriptomic Analyses. Front Microbiol. 9:2563. doi: 10.3389/fmicb.2018.02563.

Ng WV, Ciufo SA, Smith TM, Bumgarner RE, Baskin D, Faust J, Hall B, Loretz C, Seto J and Slagel J (1998) Snapshot of a large dynamic replicon in a halophilic archaeon: megaplasmid or minichromosome? Genome Research 8:1131-1141. [PubMed: 9847077]

Ng WV, Kennedy SP, Mahairas GG, Berquist B, Pan M, Shukla HD, Lasky SR, Baliga NS, Thorsson V, Sbrogna J, Swartzell S, Weir D, Hall J, Dahl TA, Welti R, Goo YA, Leithauser B, Keller K, Cruz R, Danson MJ, Hough DW, Maddocks DG, Jablonski PE, Krebs MP, Angevine CM, Dale H, Isenbarger TA, Peck RF, Pohlschroder M, Spudich JL, Jung K-H, Alam M, Freitas T, Hou S, Daniels CJ, Dennis PP, Omer AD, Ebhardt H, Lowe TM, Liang P, Riley M, Hood L and DasSarma S (2000) Genome sequence of Halobacterium species NRC-1. Proceedings of the National Academy of Sciences 97:12176-12181.

Noffke N, Christian D, Wacey D and Hazen RM (2013) Microbially induced sedimentary structures recording an ancient ecosystem in the ca. 3.48 billion-year-old Dresser Formation, Pilbara, Western Australia. Astrobiology 13:1103-1124. [PubMed: 24205812]

Peck RF, DasSarma S and Krebs MP (2000) Homologous gene knockout in the archaeon Halobacterium salinarum with ura3 as a counterselectable marker. Molecular Microbiology 35:667-676. [PubMed: 10672188]

Peck RF, Echavarri-Erasun C, Johnson EA, Ng WV, Kennedy SP, Hood L, DasSarma S and Krebs MP (2001) brp and blh are required for synthesis of the retinal cofactor of bacteriorhodopsin in Halobacterium salinarum. Journal of Biological Chemistry 276:5739-5744. [PubMed: 11092896]

Reid I, Sparks W, Lubow S, McGrath M, Livio M, Valenti J, Sowers K, Shukla H, MacAuley S, Miller T, Suvanasuthi R, Belas R, Colman A, Robb FT, DasSarma P, Müller JA, Coker JA, Cavicchioli R, Chen F and DasSarma S (2006) Terrestrial models for extraterrestrial life: methanogens and halophiles at Martian temperatures. International Journal of Astrobiology 5:89-97.

Roberge A and Moustakas LA (2018) The Large Ultraviolet/Optical/Infrared Surveyor. Nature Astronomy 2:605.

Rodrigo-Baños M, Garbayo I, Vílchez C, Bonete MJ and Martínez-Espinosa RM (2015) Carotenoids from Haloarchaea and Their Potential in Biotechnology. Marine Drugs. 13:5508-5532. doi: 10.3390/md13095508. [PubMed: 26308012] 
Rothschild LJ and Mancinelli RL (2001) Life in extreme environments. Nature 409:1092. [PubMed: 11234023]

Schwieterman EW, Cockell CS and Meadows VS (2015) Nonphotosynthetic pigments as potential biosignatures. Astrobiology 15:341-361. [PubMed: 25941875]

Schwieterman EW, Kiang NY, Parenteau MN, Harman CE, DasSarma S, Fisher TM, Arney GN, Hartnett HE, Reinhard CT and Olson SL (2018) Exoplanet biosignatures: A review of remotely detectable signs of life. Astrobiology 18:663-708. [PubMed: 29727196]

Seager S, Turner EL, Schafer J and Ford EB (2005) Vegetation's red edge: a possible spectroscopic biosignature of extraterrestrial plants. Astrobiology 5:372-390. [PubMed: 15941381]

Söll D and Rajbhandary UL (2006) The genetic code-Thawing the 'frozen accident'. Journal of Biosciences 31:459-463. [PubMed: 17206066]

Sorokin DY, Makarova KS, Abbas B, Ferrer M, Golyshin PN, Galinski EA, Ciordia S, Mena MC, Merkel AY, Wolf YI, van Loosdrecht MCM and Koonin EV (2019) Discovery of extremely halophilic, methyl-reducing euryarchaea provides insights into the evolutionary origin of methanogenesis. Nature Microbiol. 2:17081. doi: 10.1038/nmicrobiol.2017.81.

Stoeckenius W and Bogomolni RA (1982) Bacteriorhodopsin and related pigments of halobacteria. Annual Review of Biochemistry 51:587-616.

Sumper M, Reitmeier H and Oesterhelt D (1976) Biosynthesis of the purple membrane of halobacteria. Angewandte Chemie International Edition in English 15:187-194.

Walker SI, Bains W, Cronin L, DasSarma S, Danielache S, Domagal-Goldman S, Kacar B, Kiang NY, Lenardic A and Reinhard CT (2018) Exoplanet biosignatures: future directions. Astrobiology 18:779-824. [PubMed: 29938538]

Wang G, Kennedy SP, Fasiludeen S, Rensing C and DasSarma S (2004) Arsenic resistance in Halobacterium sp. strain NRC-1 examined by using an improved gene knockout system. Journal of bacteriology 186:3187-3194. [PubMed: 15126481]

Yang Y, Yatsunami R, Ando A, Miyoko N, Fukui T, Takaichi S and Nakamura S (2015) Complete biosynthetic pathway of the C50 carotenoid bacterioruberin from lycopene in the extremely halophilic archaeon Haloarcula japonica. Journal of Bacteriology JB. 02523-02514.

Yang CF, Kim JM, Molinari E and DasSarma S (1996) Genetic and topological analyses of the bop promoter of Halobacterium halobium: stimulation by DNA supercoiling and non-B-DNA structure. J Bacteriol. 178:840-845. [PubMed: 8550521]

Yim KJ, Kwon J, Cha IT, Oh KS, Song HS, Lee HW, Rhee JK, Song EJ, Rho JR, Seo ML, Choi JS, Choi HJ, Lee SJ, Nam YD and Roh SW (2015) Occurrence of viable, red-pigmentedhaloarchaea in the plumage of captive flamingoes. Scientific Reports. 5:16425. doi: 10.1038/srep16425. [PubMed: 26553382] 


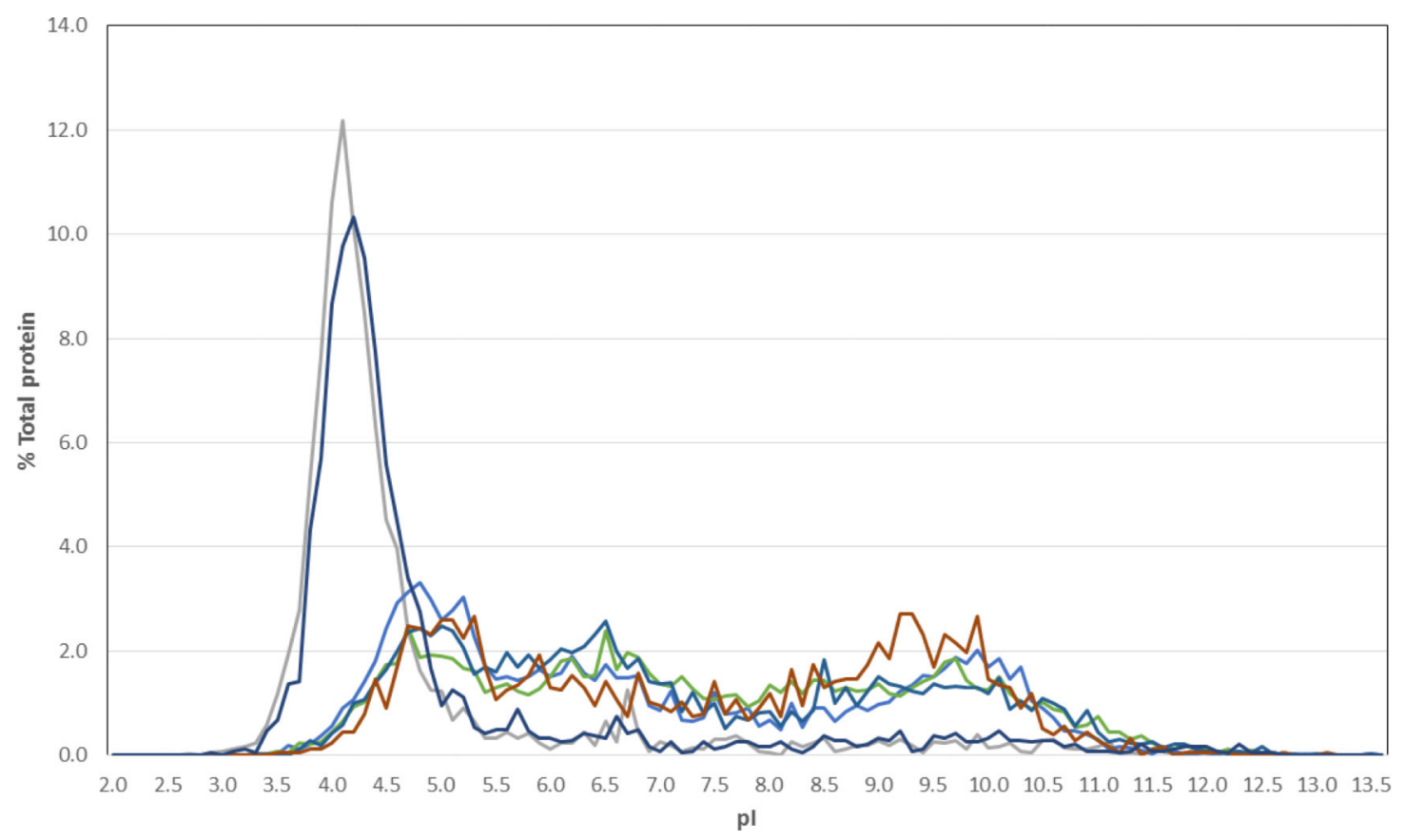

Fig. 1. Isoelectric point distribution of haloarchaeal and non-haloarchaeal proteins. Percent of total proteins with $\mathrm{pI}$ values are plotted versus pI for the Haloarchaea, Halobacterium sp. NRC-1 (gray) and Halorubrum lacusprofundi (dark blue), and nonhalophiles, E. coli (medium blue), Bacillus subtilis (light blue), Methanocaldococcus jannaschii (red) and Schizosaccharomyces pombe (green) (DasSarma and DasSarma 2015;Kennedy, et al. 2001). 


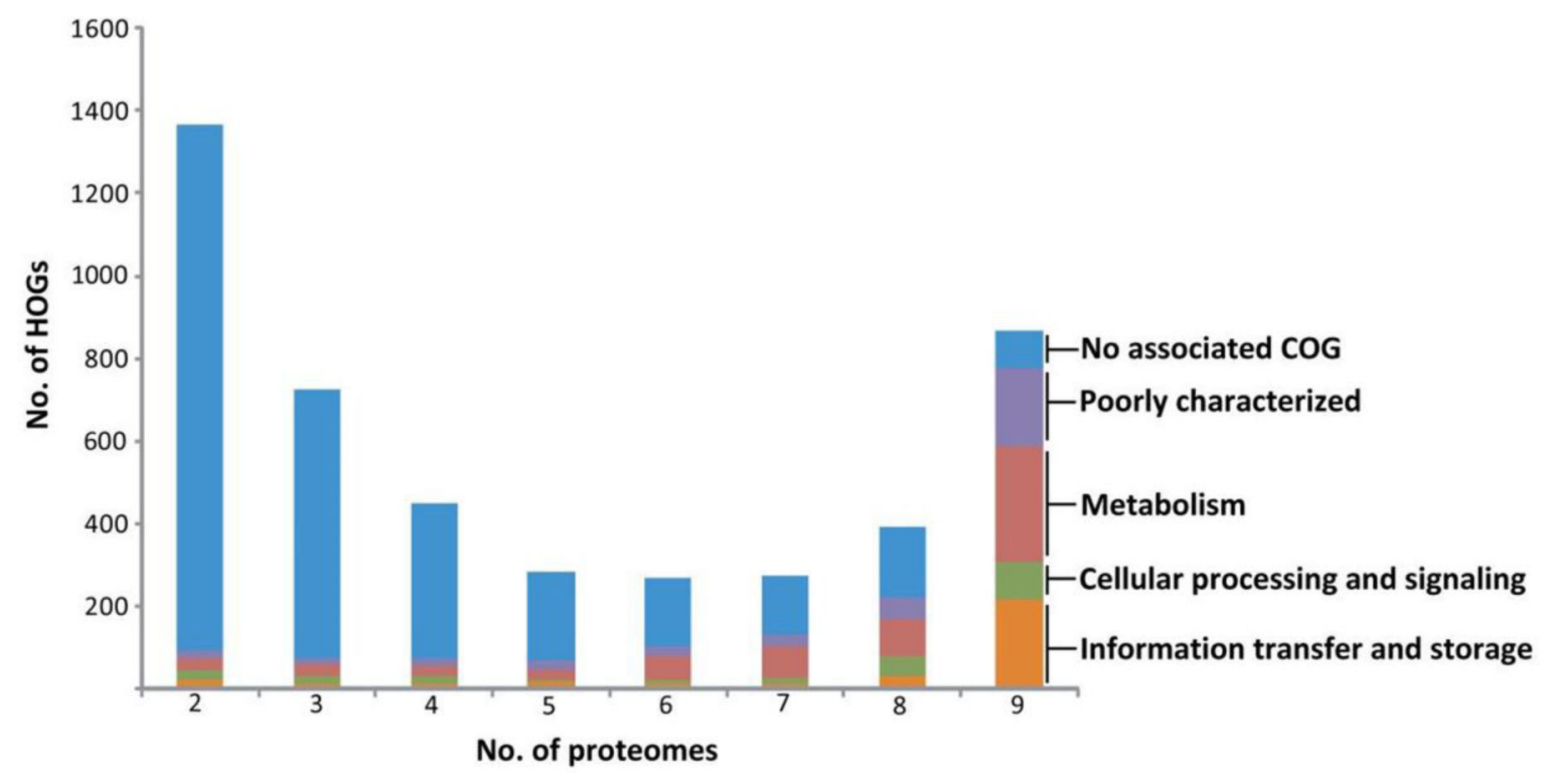

Fig. 2. Distribution of haloarchaeal orthologous groups.

Haloarchaeal orthologous groups (HOGs) identified by reciprocal Blast analysis exhibit a bimodal distribution, with the largest number of proteins with unknown function and no associated Clusters of Orthologous Groups (COGs) shown in blue. Those with known or predictable function present in haloarchaeal proteomes are shown in other colors (orange, green, reddish brown, and purple) with functions indicated on the right (Capes, et al. 2012). 


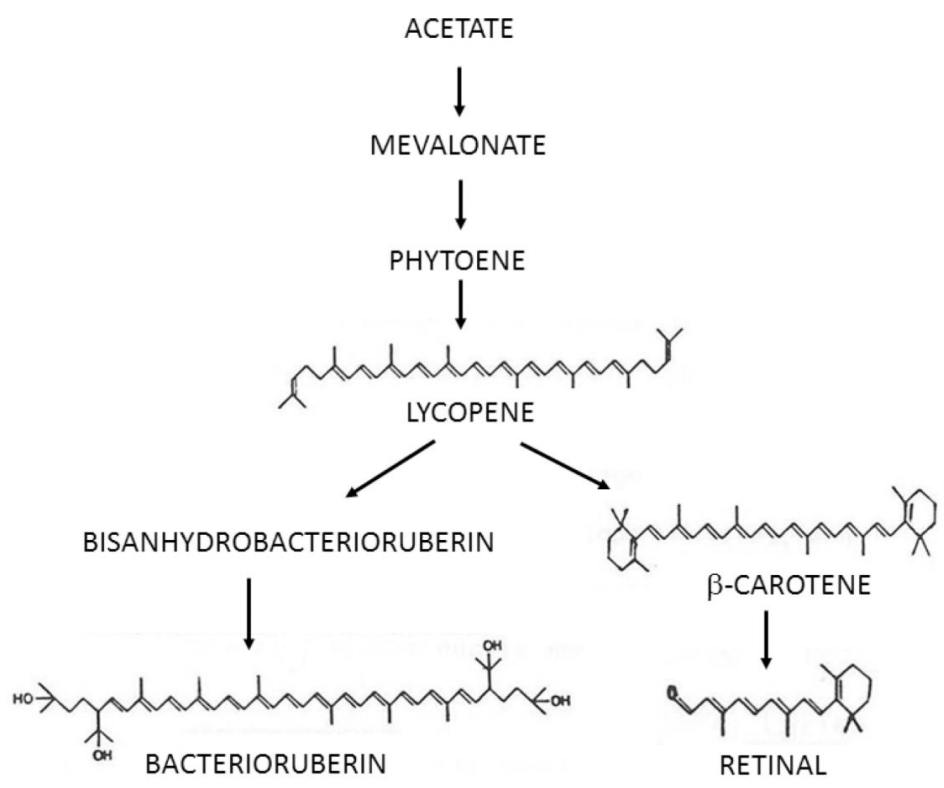

\section{Photoprotection and repair Phototrophic growth}

Fig. 3. Pigment biosynthesis in Haloarchaea.

Biosynthesis of common pigments in Haloarchaea such as Halobacterium species proceeds from acetate via mevalonate to lycopene where the pathway splits to form the bacterioruberins or $\beta$-carotene and retinal. Bacterioruberins have been implicated in photoprotection and repair while retinal acts as the chromophore in purple membrane used for phototrophic growth (DasSarma, et al. 2001). 


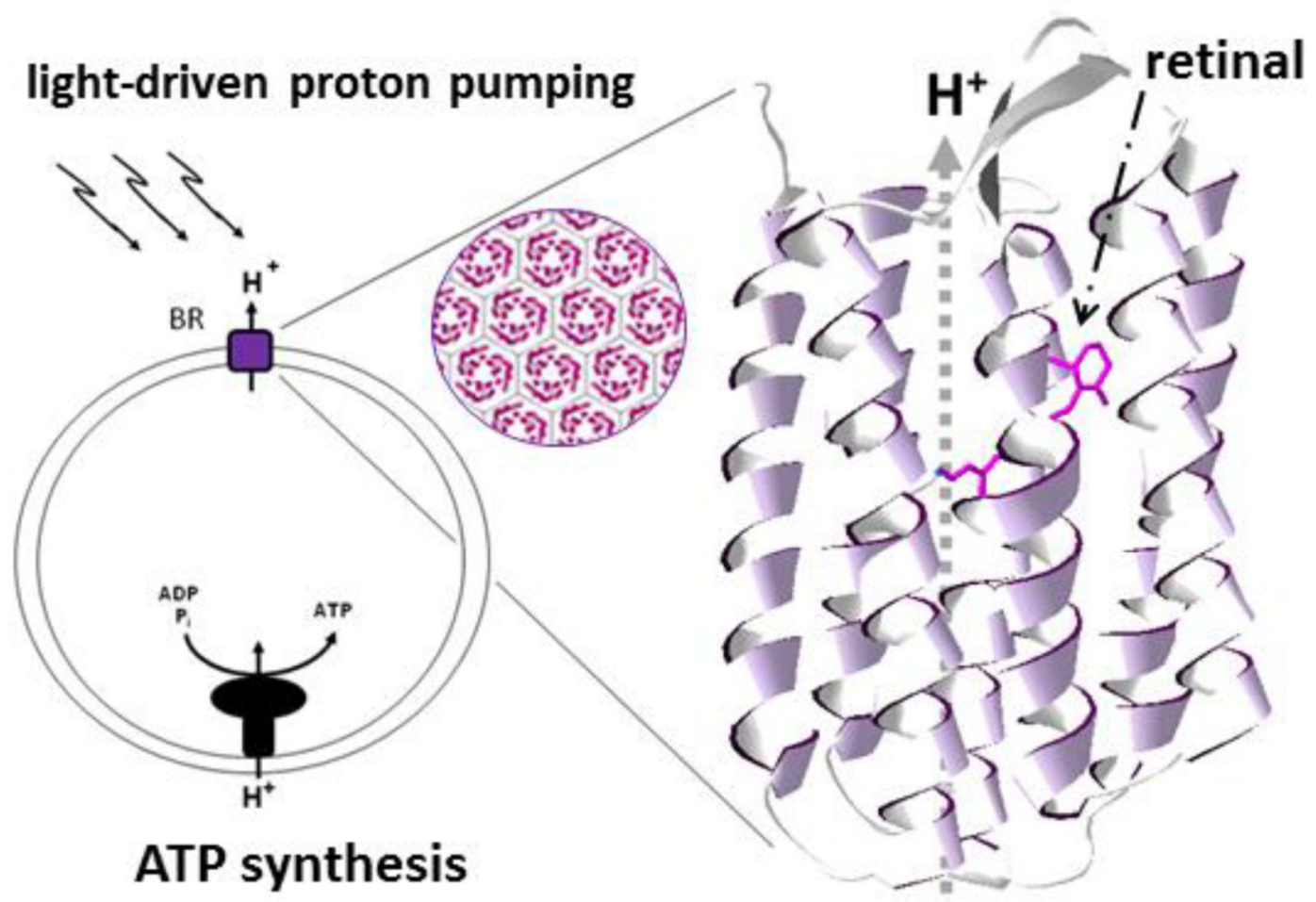

Fig. 4. Phototrophic capability of Haloarchaea.

Haloarchaea such as Halobacterium sp. NRC-1 contain the retinal protein bacteriorhodopsin (BR) which is an outwardly directed light-driven proton pump producing a proton-motive gradient that drives ATP synthesis using ADP and $\mathrm{P}_{\mathrm{i}}$ (left). Trimers of BR form a hexagonal lattice in the membrane (circular inset, center). BR's seven transmembrane a-helical segments are shown in ribbon form (right) with the bound retinal (stick structure) involved in promoting transport of protons $\left(\mathrm{H}^{+}\right.$ions) across the membrane (DasSarma and Schwieterman 2019). 

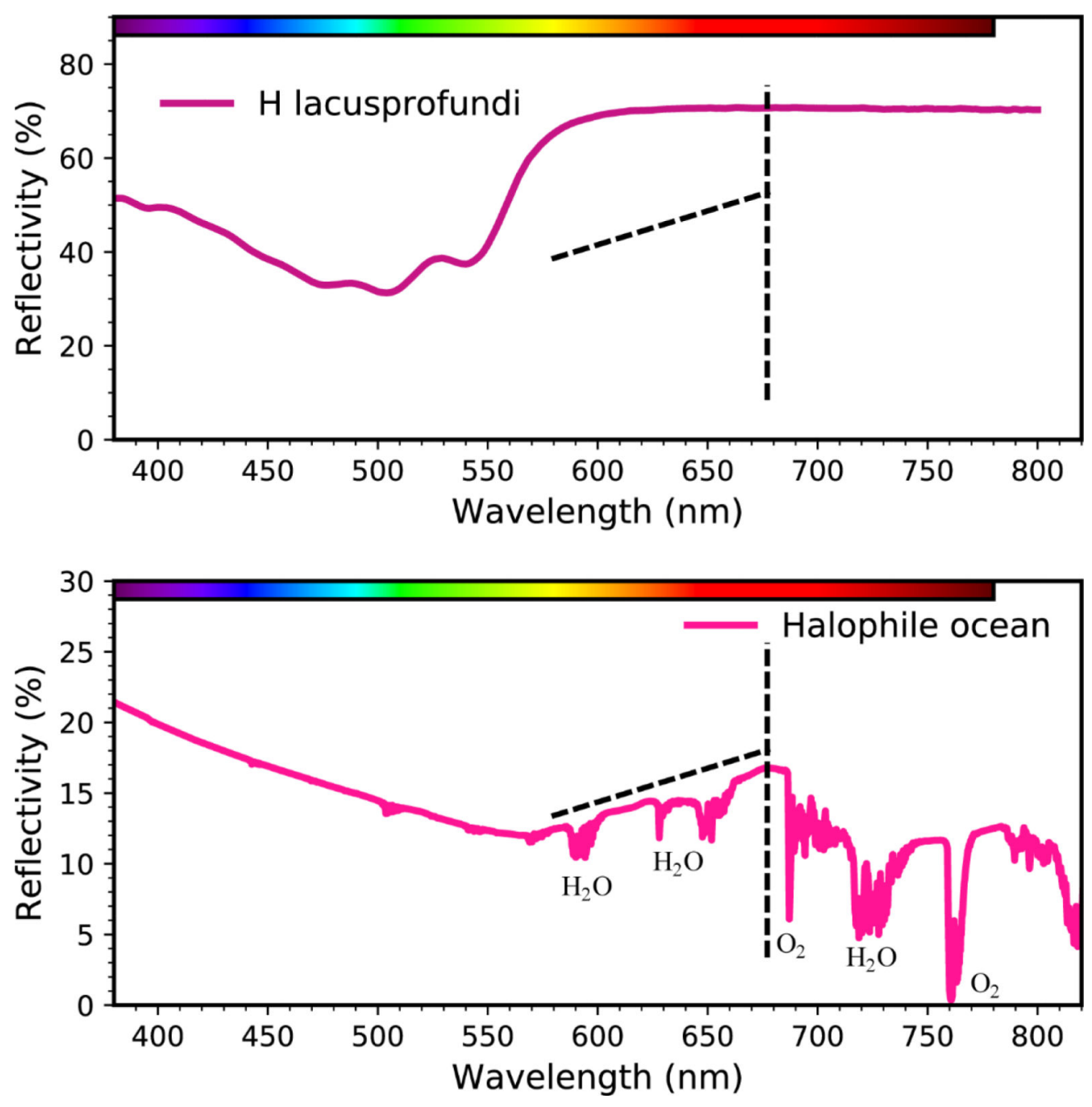

Fig. 5. Reflective green edge from haloarchaeal pigments.

A laboratory reflection spectrum of $H$. lacusprofundi (top panel) and an environmentally characterized halophile bloom under a simulated Earth atmosphere (bottom panel), including atmospheric absorption and scattering, are shown, plotting wavelength in nanometers (nm) (abscissa) versus percent reflectivity (ordinate) (DasSarma and Schwieterman 2019). Dashed lines are shown for reference and strong water $\left(\mathrm{H}_{2} \mathrm{O}\right)$ and oxygen $\left(\mathrm{O}_{2}\right)$ interference bands labeled. 
Table 1.

Examples of extreme conditions tolerated by Haloarchaea

\begin{tabular}{lll}
\hline Extreme & Species & Environment \\
\hline Salinity & Halobacterium species & Grows in saturated (>30\%) NaCl, e.g. in saltern crystallizers \\
Cold & Halorubrum lacusprofundi & Grows at low perennial temperatures to $-18^{\circ} \mathrm{C}$ e.g., in Deep Lake, Antarctica \\
Heat & Halorhabdus tiamatea & Grows at elevated temperatures to $60^{\circ} \mathrm{C}$, e.g. in deep sea brine pool, Red Sea \\
Oxygen-free & Halobacterium and Haloferax species & Grow anaerobically with alternate electron acceptors, e.g., nitrate, perchlorate \\
Desiccation & Halobacterium species & Survives in subsurface brine inclusions, e.g. in Permian salt deposits \\
Ionizing radiation & Halobacterium species & Survives ionizing radiation $8-11$ kGy from LINAC and X-rays in laboratory \\
UV radiation & Halobacterium species & Survives exposure to 70 Jm ${ }^{-2}$ UV-C radiation, e.g, in stratosphere \\
Low pressure & Halorubrum lacusprofundi, Haloarcula & Tolerant of extremely low pressures, in stratosphere and space \\
& 'G' & \\
Toxic ions & Halobacterium species & Tolerant of arsenic, magnesium, and perchlorate \\
Alkalinity & Natronomonas pharaonis & Grows at alkaline pH (8.5-9.0), e.g. in Lake Magadi, Kenya \\
Acidity & Halarchaeum acidiphilum & Grows at acidic pH (4.0-6.0), isolated from commercial salt \\
\hline
\end{tabular}

Extremophiles. Author manuscript; available in PMC 2021 January 01. 